\title{
Structural Self-organized Holonic Multi-Agent Manufacturing Systems
}

\author{
José Barbosa ${ }^{1,3,4}$, Paulo Leitão ${ }^{1,2}$, Emmanuel Adam ${ }^{3,5}$, Damien Trentesaux ${ }^{3,4}$ \\ ${ }^{1}$ Polytechnic Institute of Bragança, Campus Sta. Apolónia, \\ Apartado 1134, 5301-857 Bragança, Portugal \\ ${ }^{2}$ LIACC - Artificial Intelligence and Computer Science Laboratory, R. Campo Alegre 102, \\ 4169-007 Porto, Portugal \\ ${ }^{3}$ Univ. Lille Nord de France, F-59000 Lille, France \\ ${ }^{4}$ UVHC, TEMPO research center, F-59313 Valenciennes, France \\ ${ }^{5}$ UVHC, LAMIH, F-59313 Valenciennes, France \\ (e-mail: $\{$ jbarbosa,pleitao\}@ipb.pt; \\ \{emmanuel.adam, damien.trentesaux\}@univ-valenciennes.fr)
}

\begin{abstract}
Worldwide systems are increasingly growing into unprecedented complexity levels. This increase of system complexity has to be tackled with new control approaches, where decentralization is playing an important role and particularly the Multi-Agent Systems and Holonic Systems. Despite the benefits of this distribution, new problems arise such as the need for entities coordination. This paper proposes an innovative holonic multi-agent system architecture, named $\mathrm{ADACOR}^{2}$, which sets foundation on the already proved ADACOR architecture. This new control architecture is empowered by a two vector selforganization, called behavioural and structural self-organization. This paper describes the structural self-organization vector, particularizing the need for embedding in it learning techniques and nervousness stabilizer. A futuristic test bed, inspired in a real flexible-manufacturing system, is used to demonstrate the benefits of this vector in the architecture.
\end{abstract}

Keywords: self-organization, multi-agent systems, reconfigurable manufacturing control

\section{Introduction}

Nowadays, problems' complexity has no parallel in past since the world complexity has grown into unprecedented levels. As examples, in avionics the system complexity has grown from the first airplanes to the nowadays state of the art airplanes, such as the Airbus A380, or in economics where an evolution from local economy to worldwide global markets has noticed. Complexity can also be found on diverse fields of modern society and range from telecommunications to economics or from health to ecosystems in nature. This amazing level of system complexity makes urgent the development of new solutions, methodologies and approaches to better tackle the

adfa, p. 1, 2011.

(C) Springer-Verlag Berlin Heidelberg 2011 
current demands, such as controlling the chaotic behaviour that these systems may display. Also important to consider is to manage the butterfly effect present in such systems, where small changes in one part of the system can have a huge impact on the other side or at medium/long term. Manufacturing systems domain is also increasingly becoming more complex where a multitude of unexpected or unpredicted events are constantly appearing, such as rush orders, resources malfunctions, late changes requests, order cancelation/modification.

The classical solution to solve these problems passes by the use of information and communication technologies and high processing power, usually using a centralized structure, where decision-making functions are centralized in one node that guarantees high levels of performance optimization. This is currently true for most of the control systems and in fact this approach still works pretty well if one assumes that the system is perfect in the sense that it doesn't have unexpected events. But, the truth is that nowadays industrial systems must deal daily with unexpected events and in that sense must promptly respond, effectively, to them. In this way, these traditional centralized approaches are unable to properly cope with these requirements due to its rigid structure.

One of the most consensual solutions to handle very dynamic and complex systems is the distribution of the processing capacity throughout the system by giving to individuals, more or less intelligent, the capacity to manage its local action space. In this approach, the global system's goals are achieved by cooperation, e.g. by means of information sharing or by the association of individual skills.

Several paradigms have risen to address these challenges, proposing distributed approaches, e.g. Multi-Agent Systems (MAS) and Holonic Systems (HS). MAS [1] derive from the artificial intelligence field and is built upon autonomous and cooperative entities called agents, which are intelligent and autonomous entities capable to take decisions based on local knowledge. HS got inspiration by the work of A. Koestler [2] and has several differences from the MAS such as the creation of stable intermediary states as a mean to achieve optimization or the notion of holarchy which is a mixture of heterarchical autonomous entities (like in MAS) and hierarchical organizations, possibly considering the use of recursivity. The elementary concept in a HS is the term holon, proposed by Koestler, which derives from the Greek word holos which means whole and the suffix on that suggests a part like in proton. Another key difference between HS and MAS is that a holon can easily be designed as the integration of a physical with an informational part, facilitating the design of more real time and control-based approaches.

Detailing into manufacturing systems, these two paradigms, particularly MAS applications, have been used in the past in several different applications (see for example the surveys [3, 4]). One example, of a Holonic Manufacturing System, is the PROSA reference architecture, which defines the basic holons that a manufacturing system must have as also their basic interactions. Other example is the ADACOR (ADAptive holonic COntrol aRchitecture for distributed manufacturing systems) architecture [5], that proposes an adaptive production control system that switches from a stable state, where the system is organized in a hierarchical approach to achieve optimization, to transient state, where the control is passed into the lower 
level entities for a prompt response to disturbances. This binary state has shown very good results facing very condition changing environments, but restrings the system to the two pre-defined states.

The current work sets foundation on this already proven holonic system and unleashes it from these two states by allowing the system to dynamically evolve without any pre-defined boundaries. This evolution, called $\mathrm{ADACOR}^{2}$, is supported by the self-organization concept, found in biology and other fields, decoupled in two different levels: behavioural and structural self-organization. The first one, appears at the micro-level, and is related to the increase of the performance of the individual holons by means of proper adaptation of their behaviour. The second one, described in this paper, appears at the macro-level, and is about the dynamic structural organization of the holons. Having this mechanism in mind, it can be stated that the ADACOR evolved into a more dynamic and open structure allowing the re-arrangement of the entities in the system, exploring the unpredictability of complex systems.

In such dynamic self-organized systems, the problem of nervousness [6-8], which is related to the will that entities have to change at the minimum stimuli from external or internal events, should be considered to guarantee the system stability. Additionally, the development of structural self-organized systems must consider important questions, like what is the best structural configuration, when the system should evolve/re-configure, and how to evolve, which answers may consider the implementation of learning mechanisms.

This paper depicts the architecture for the structural self-organization, as part of the self-organization principle used within the $\mathrm{ADACOR}^{2}$ approach. To fully demonstrate the proposed approach, the architecture was implemented, tested and validated using a Flexible Manufacturing System (FMS) that is a re-design of the real AIP-PRIMECA, located at the of the Universite de Valenciennes et du Hainaut-Cambrésis, in which the resources have moving capabilities.

The rest of the paper is organized as follows: Section 2 overviews the selforganization concept defined in the $\mathrm{ADACOR}^{2}$ holonic multi-agent system architecture and Section 3 details one of its vectors, the structural self-organization. Section 4 describes the internal architecture of a holon belonging to $\mathrm{ADACOR}^{2}$ focusing the model to support the self-organization capabilities. Section 5 presents the experimental validation of the proposed approach by using the AIP PRIMECA flexible manufacturing system. At last, Section 6 rounds up the paper with the conclusions.

\section{$2 \quad$ ADACOR ${ }^{2}$ : enhanced by self-organization}

The ADACOR control architecture defines four types of holons [5]: Supervisor Holon $(\mathrm{SH})$, Product Holon $(\mathrm{PH})$, Task Holon $(\mathrm{TH})$ and Operational Holon $(\mathrm{OH})$. Besides defining the roles, behaviours and interactions among these holons, ADACOR also introduced an adaptive production control based on balancing the control structure between a stationary state (where the holons are organized according to a hierarchical structure) and a transient state (where the holons are organized in a complete heterarchical structure). The stationary state should be used as much as possible to achieve 
optimization, and the transient state should be adopted in presence of perturbations to provide adaptive response (returning to the stationary state after the resolution of the perturbation, which should be as fast as possible).

This switching mechanism proved to be very efficient [5] to manage the production control in very unpredictable scenarios, but is restrained to the two pre-defined states. $\mathrm{ADACOR}^{2}$ unleashes the architecture from these chains by allowing the system to dynamically evolve throughout a set of configurations, dynamically discovered, using the concept of self-organization usually found in biology.

The self-organization mechanism considered in $\mathrm{ADACOR}^{2}$ comprises two vectors, as illustrated in Fig. 1: the first vector, to cope with smaller impact perturbations, called behavioural self-organization, is achieved by providing holons a set of different behaviours that are dynamically selected accordingly to the current needs. The second vector, called structural self-organization, is more related to dynamic changing of the relations and interactions among holons. Additionally, since $\mathrm{ADACOR}^{2}$ follows the holonics principles, the structural self-organization is also related to the establishment of intermediary states e.g., the group formation. This macro-level self-organization definition allows the system to dynamically adapt, in a more drastic manner, to larger perturbations by changing the holons relations.

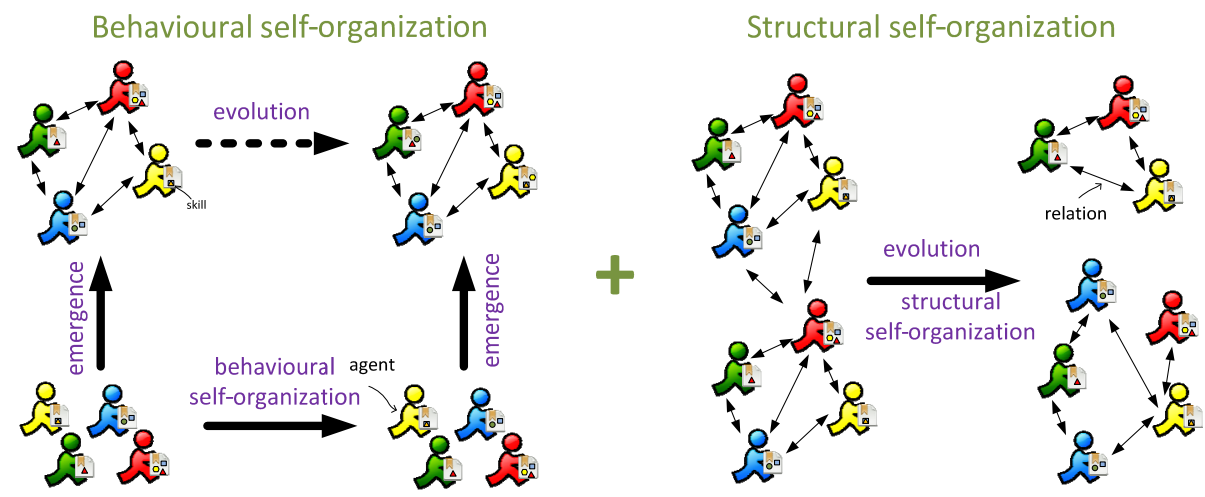

Fig. 1. Self-organization in $\mathrm{ADACOR}^{2}$ holonic architecture

In this way, a configuration is defined as a snapshot of the system at a given point in time, having information of the holons state, behaviours and their relations (representing the organizational structure of the system). This context-aware knowledge is helpful in situations where the system must evolve, in the way that holons reason on this set of configurations to learn from past situations. To achieve this, ontologies plays an important role enabling the sharing of knowledge in this dynamic and distributed system. In this way, besides the ADACOR manufacturing ontology [9] that has already been developed, a complementary ontology is developed to support the sharing of knowledge related to the configuration contextualization in this dynamic, distributed system. In case of conditions change, the holon(s) that have recognized the situation can find a starting point from similar conditions through the ontology rea- 
soning. This warm, i.e. not being executed from scratch, self-organization allows a faster and most optimized way to evolve into a stable configuration.

The decision of how to better cope with the condition changes, considers learning mechanisms aggregated to a nervousness mechanism to select the best option to take from either behavioural or structural self-organization (pushing the system into the limits but remaining under control). This selection is accomplished by analysing the performance indicators output from the known self-organization mechanisms of the holon. Additionally, learning mechanisms are also responsible to detect new opportunities to evolve, making possible for the system to increase its performance even if on the absence of a disturbing event.

Resuming, the main difference between $\mathrm{ADACOR}^{2}$ and its predecessor is the removal of the two pre-defined states by introducing the concept of self-organization spited at two levels: behavioural at micro level and structural at macro-level.

Next sections will detail the structural self-organization mechanisms.

\section{Structural self-organization in ADACOR ${ }^{2}$}

In this work, the structural self-organization is classified in a three level division. This classification closes the gap with the behavioural self-organization by relating it with the structural self-organization.

- Level 0 (emergence): the relations between holons are changed as consequence of the behavioural self-organization. The holon by changing its internal behaviour could, indirectly, imply changing its external relations. This level of structural changing is classified as weak since the structural change is not driven directly by the need.

- Level 1 (logical structural self-organization): each holon is always trying to optimize its place into the holarchy structure. This constant optimization may drive the holon to change from holarchy, to belong to several holarchies at the same time or as freelancer to work completely autonomously.

- Level 2 (physical structural re-organization): similar to level 1 with the addition that holons, e.g. OHs, can physically change their place, changing not only their relations and positions in the holarchy but their physical places.

The stimulus that can trigger a structural self-organization can be any event that disrupts or deviates the predicted function of the system, such as a rush-order, order cancellation, production quality issue, supply shortage, or resource malfunction. To illustrate this, two examples can be provided. The first example of a stimulus that can start a structural self-organization is the introduction of a big batch order. Let's assume that the system is functioning in a stable state with a given configuration, and that at a given point in time, a very large order arrives into the system. The system, realizing these new constraints, will select the most adequate structure to better cope with this. In the simplest way, individual holons can adjust themselves by changing their behaviour or if the impact to the system is large, the holons may find the need to start a structure re-organization. In other words, the holons can change relations 
keeping the holarchies composition, holons can change holarchies or, if possible, the physical resources can move into another working position to improve the performance indicators.

A second example, at a longer time window, concerns the smoothed changing in a usual set of recurrent orders. Let consider two types of products, A and B. Let also consider that a customer asking for a specific kind of product type B takes more and more importance compared to historical customers asking for product type A. This evolution in the production demand can be detected and the re-arrangement of the structure occurs smoothly to evolve with the needs of customers from a production optimized to make product of type A to a production optimized to make products of type B.

A pertinent question is how does the $\mathrm{ADACOR}^{2}$ holons self-reorganize structurally? Getting inspiration from the social behaviour of swarms of fishes and birds, it is concluded that they function very well as a group, maintaining system equilibrium and, as a group, avoid predators (in our case, these are the system external perturbations). What is more amazing is that this is achieved by following very simple rules and without any central authority in charge.

$\mathrm{ADACOR}^{2}$ holons follow the same basic principles as swarms, trying to constantly make cohesive groups but maintaining distances and having crowd management. One observed principle that is not followed in $\mathrm{ADCOR}^{2}$ is the lack of central authority. In fact, a central authority is used to create stable intermediary states into the holonic holarchy introducing high levels of optimization. These higher level holons, i.e. SHs, have under their supervision a set of $\mathrm{OHs}$, which they try to optimize. Another atomic rule in structural self-organization, under the SH umbrella, is to endow each group with a set of services, as diverse as possible, opening the possibility to attend a wider set of requests. This rule is not restrictive, allowing making more dedicated groups, i.e. more skilled groups, and also makes the system less dependent of a limited group of holons. This characteristic can be seen in Fig. 2, where the services are distributed uniformly among groups.

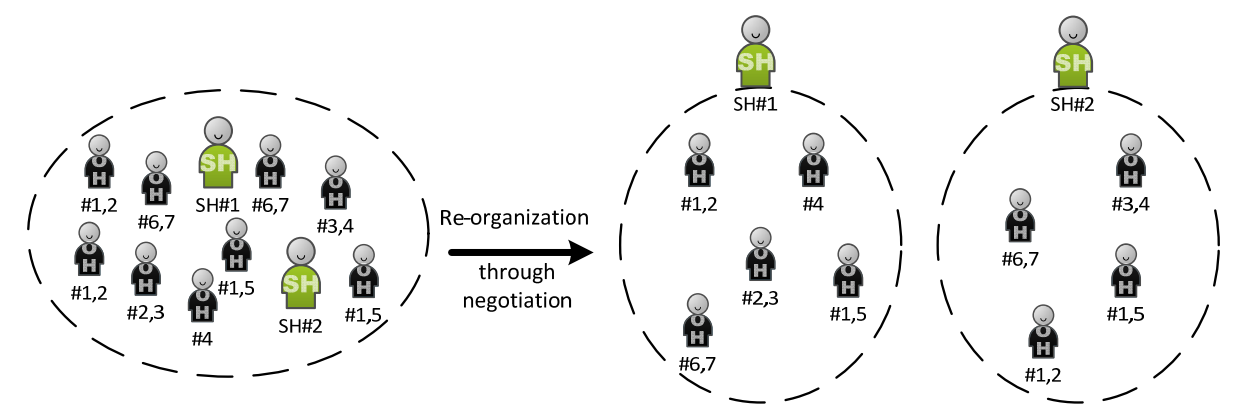

Fig. 2. Equality services distribution in $\mathrm{ADACOR}^{2}$ group formation

Another crucial rule is related to the possibility of an $\mathrm{OH}$ be shared between SHs. Let's suppose that a given service is missing under a stable state governed by a $\mathrm{SH}$. At a given point in time, a SH may find the need to acquire one more service in order 
to respond accordingly to an external demand or even to the malfunction or decommissioning of the existing $\mathrm{OH}$ that offers that service. In this case, the SH will search within the system domain for OHs that offers the same service. Once found, the $\mathrm{SH}$ will start a negotiation process, with the other $\mathrm{SH}$ or directly with the $\mathrm{OH}$ in case of no hierarchical dependency, to find equilibrium between these two groups, i.e. not degrading heavily the service from the source group but allowing the second one to have the possibility to acquire that service. In the case of $\mathrm{OH}$ sharing among SHs, its utilization is achieved by the higher level negotiation of the SHs to whom he belongs. An example of this mechanism is depicted in Fig. 3 where the $\mathrm{OH}$ that offered the service \#4 becomes unavailable, triggering the search to re-acquire the lost service. After a negotiation process (i.e., the first agreement from the SHs level), the SH\#1 proposes the $\mathrm{OH}$ that has the desired service to belong also to its group. If agree, the holons start the structural re-arrangement and relationship redefinition ending on the holon sharing among groups.

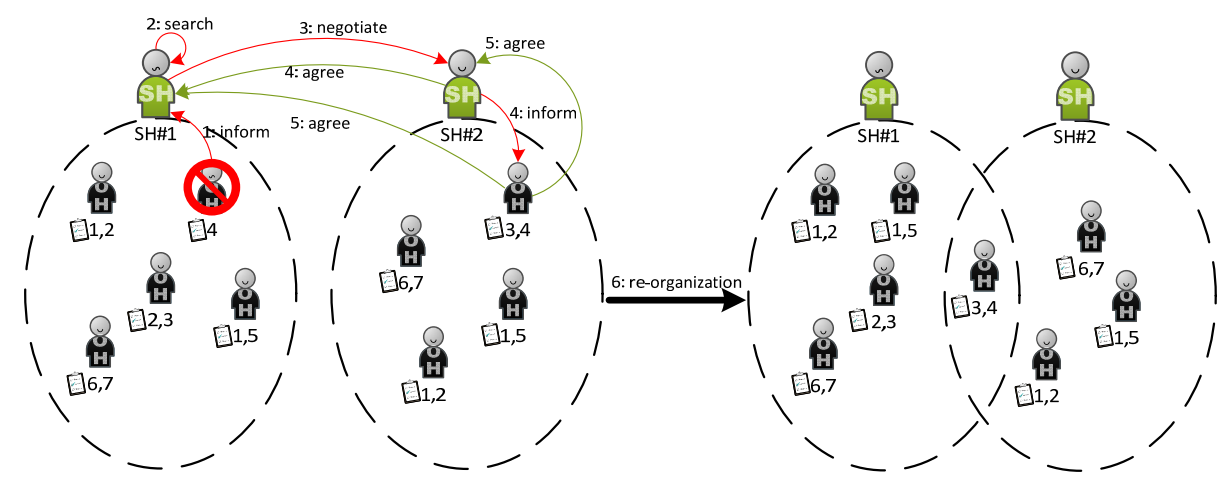

Fig. 3. Structural self-organization initiation: service disruption

Naturally, ADACOR ${ }^{2}$ being a holonic multi-agent system allows that this negotiation process may be started directly with the $\mathrm{OH}$, bypassing the $\mathrm{SH}$.

\section{Architecture of individual holons to support structural self- organization}

The structural self-organization of the system is driven by the behaviour of the distributed holons that constitutes the $\mathrm{ADACOR}^{2}$ system. For this purpose, each one exhibits proper mechanisms in its internal structure architecture to support the structural self-organization mechanism. Fig. 4 illustrates the internal architecture of a holon belonging to $\mathrm{ADACOR}^{2}$, focusing mainly the model to support self-organization, which comprises primarily the monitoring, discovery, reasoning, nervousness stabilizer and dispatcher components.

The structural self-organization process can be very time-consuming, especially in large-scale systems. In this way, $\mathrm{ADACOR}^{2}$ has on the start of this process a contextaware feature. The system, at every disrupting event, takes a snapshot of the system 
state additionally with the actions taken and the achieved results (i.e., performance indicators). This context-aware mechanism, aided with data processing, e.g. data mining, will facilitate futures events by allowing the system to filter from the best measures taken to similar events and start a warm structural self-organization.

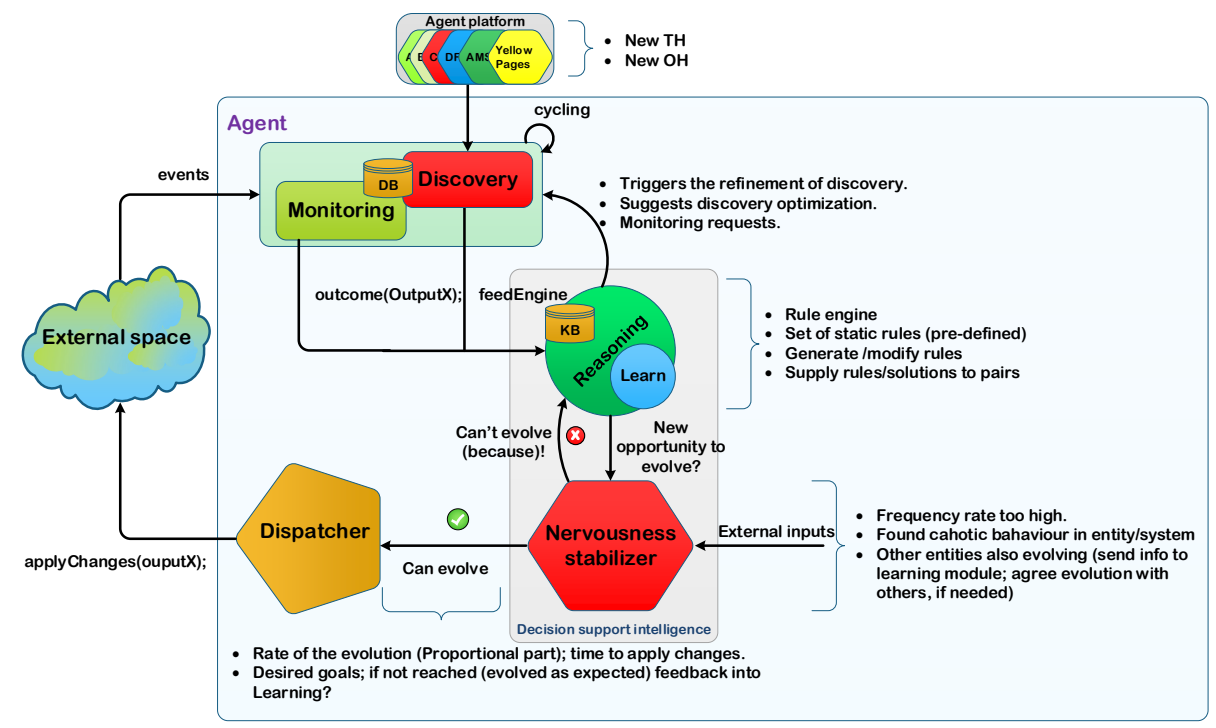

Fig. 4. Model to support the self-organization mechanisms

The discovery and monitoring modules, supported by a knowledge base, will feed the reasoning and learning engines that are responsible to discover new opportunities to evolve and decide how to evolve. In this approach, the learning capabilities embed in holons assume crucial importance to support the generation of new knowledge, contributing to behave better in the future. The implementation of such learning capabilities can range from simple observation or repetition techniques to more complex ones, such as neural networks or support vector machines. The selection of the learning techniques to be deployed in $\mathrm{ADACOR}^{2}$ must consider several features. Among them are the type of communication to be considered (direct or indirect) and scalability. Other issues are related to the fact that multi-agent systems are typically deployed into dynamic environments and with problem decomposition to reduce complexity [10].

$\mathrm{ADADCOR}^{2}$ uses different types of learning techniques in different parts of holons or different parts of the system. Social learning is used to support the propagation of new behaviours among the holons or by PHs when creating THs, passing accumulated knowledge from past THs. A reinforcement learning mechanism may be used as a behaviour mechanism for route selection. This reinforcement learning mechanism, inspired in the ant food foraging mechanism, separates the exploration and exploitation phases, and relies in the random decision of the holon as a way to discover new solutions. The feedback from past decisions is also used, where bad past decisions will have a negative impact in the future choice for the same decision as opposite to a good result that will have a positive impact. 
In such dynamic environments, where holons are autonomous and self-organized, the system has the predisposition to become unstable and chaotic. Instability and chaos are addressed in $\mathrm{ADACOR}^{2}$ by applying nervousness stabilizers to each of the two self-organization mechanisms. The purpose of the nervousness stabilizers is to bring stability into the behaviour of the holons and to the structural self-organization in $\mathrm{ADACOR}^{2}$. By introducing these stabilizers, the system operates in a such manner that is pushed to its limits by enhancing the self-organization and chaotic principles, but always under control. Note that any decision to evolve provided by the reasoning and learning engines is evaluated in the nervousness stabilizer which will validate the decision.

Typical approaches to calm the holons' will of change passes by restring the number of changes during a given time, by allowing the entities to change only at predefined intervals or by setting the exploration/exploitation threshold [8]. ADACOR ${ }^{2}$ holons use an innovative technique [8] where the inspiration from the classical control theory, namely the Proportional, Integrative and Derivative (PID) controller, acts as a constrain to the response time and defines the accepted deviation from the known optimal plan or the speed of the deviation mitigation.

\section{The AIP cell application example}

The testing and validation of the proposed structural self-organization mechanism uses an adapted version of the AIP-PRIMECA manufacturing system, located at the Université de Valenciennes et du Hainaut-Cambrésis. In this scenario, resources can self-reorganize, responding in a more appropriate way to perturbations maintaining high levels of performance. This test scenario allows to verify all potentialities of the structural self-organization of ADACOR ${ }^{2}$.

\subsection{System description}

The real AIP-PRIMECA manufacturing system comprises a set of 6 resources, each one able to perform a set of services that are linked by a fixed conveying system [11].

The proposed system assumes that resources have moving capabilities in the sense that they can unplug from one working position and be moved into another working position. A working position is a place in the environment that is equipped with supporting systems for resource normal functioning, such as power and communications. The rail type conveyor system present in the real AIP cell is removed and the transportation between resources is accomplished by using Automatic Guided Vehicles (AGVs). Fig. 5 depicts the conceptual view of the futuristic cell, where the purple places represent working positions and the green ones can only be used for routing paths. For transportation calculation times it is assumed that moving from one point to another takes 3 seconds to the AGV in a clear path. The shifting of resources depends from resource to resource since the complexity of cables, size and weight can vary. 

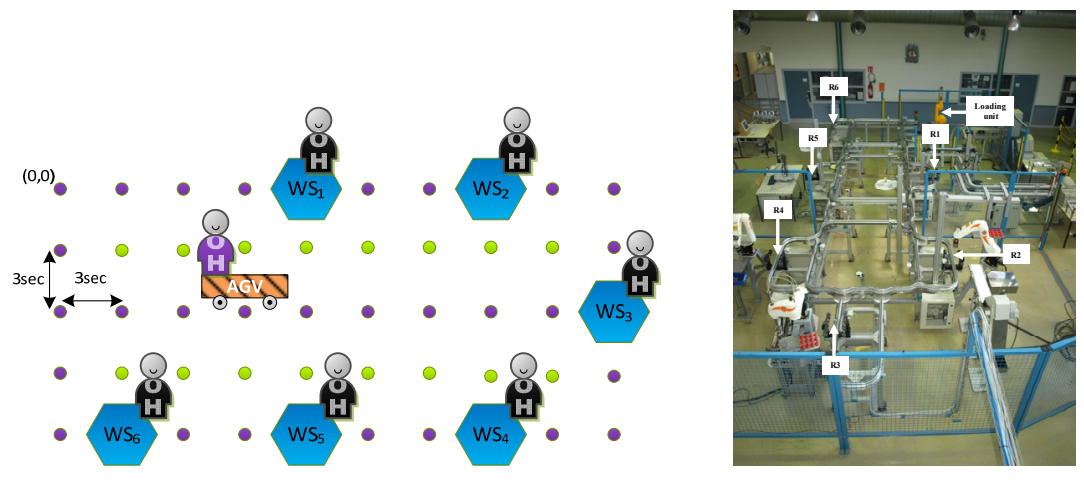

Fig. 5. Cell configuration test scenario (on the rigth side the real AIP cell).

Having this in mind, the $\mathrm{ADACOR}^{2}$ holons are deployed into the production control system, with resources being mapped as $\mathrm{OHs}$ and each $\mathrm{AGV}$ as a specialized transporter instantiation of the $\mathrm{OH}$ (see Fig. 5). This test bed is composed by 6 resources, each one having a number of offered services, as illustrated in Table 1.

Table 1. Resource service list and processing times

\begin{tabular}{c|cccccc}
\hline service & $M_{1}$ & $M_{2}$ & $M_{3}$ & $M_{4}$ & $M_{5}$ & $M_{6}$ \\
\hline Loading & 10 & & & & & \\
Unloading & 10 & & & & & \\
Axis & & 20 & 20 & & & \\
R_comp & & 20 & 20 & 20 & & \\
I_comp & & & & 20 & & \\
L_comp & & 20 & 20 & 20 & & \\
Screw_comp & & & & & & \\
Inspection & & & & & 5 & \\
Recovery & & & & & & 60 \\
\hline
\end{tabular}

A level 3 structural self-organization was used for the tests, since resources will have the possibility to shift place in the shop-floor. The structural self-organization mechanism is triggered when a batch of orders is allocated to an $\mathrm{OH}$. After receiving a batch of orders, the $\mathrm{OH}$ will start an information gathering from other $\mathrm{OHs}$, where resource queue, allocated work-orders, processing times and actual location, is exchanged. The procedure used follows the principle that the most overloaded $\mathrm{OH}$ are the most critical ones and in that way tries to allocate them first by minimizing the transportation times between them. After finishing the allocation of all $\mathrm{OHs}$, each $\mathrm{OH}$ sends the information of either better or worst solution achieved, from actual resources disposition. In the case of a better solution, the $\mathrm{OH}$ sends the KPI and the new allocation places to all the $\mathrm{OH}$. The overall best solution, found in each $\mathrm{OH}$, is automatically assumed and used.

\subsection{Analysis of the results}

The described system, developed using the JADE framework [12] and the Java Expert System Shell [13], initializes with a manufacturing order to produce 2xBELT. After 
120 seconds a new manufacturing order to produce 5xAIP appears in the system, which triggers a structural self-organization, see Fig. 6.

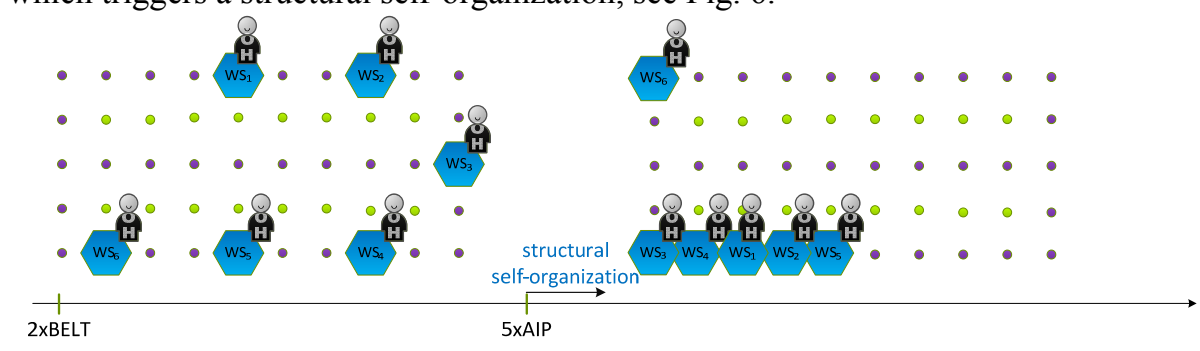

Fig. 6. Structural self-organization in practice

Two scenarios are simulated and compared to evaluate the structural selforganization. On the first scenario the structural self-organization is disabled while it's enabled on the second one. For each scenario, several simulations were done and average values are presented in Table 2 .

Table 2. Experimental results

\begin{tabular}{|c|c|c|}
\hline \multirow{4}{*}{$\begin{array}{c}\mathrm{C}_{\max } \\
\text { \%transpTime } \\
\text { avg(order) }\end{array}$} & Without structural self-organization & With structural self-organization \\
\hline & $1147,5 \mathrm{~s}$ & $1112 \mathrm{~s}$ \\
\hline & $19,4 \%$ & $14,9 \%$ \\
\hline & $725,2 \mathrm{~s}$ & $646,2 \mathrm{~s}$ \\
\hline
\end{tabular}

As can be observed by the analysis of the previous table, enabling the structural self-organization reduces the overall Cmax, allowing a performance improvement. This is mainly accomplished by the reduction of transportation times when the structural self-organization is enabled (reduction from $19,4 \%$ to $14,9 \%$ ). Also a reduction of the time to process each individual order was noticed.

The achieved preliminary experimental results show the merits of the structural self-organization approach to face severe changing conditions. However, the experiments also showed new possibilities to improve the proposed algorithm, e.g. by considering optimization in the re-configuration of the resources during the structural self-organization process.

\section{Conclusions}

System complexity has grown into unprecedented levels, turning on the need of new control architectures. In this subject, MAS and HS play an important role by proposing a distribution of the processing capacity by several autonomous entities.

This paper introduced an innovative control architecture called $\mathrm{ADACOR}^{2}$, which considers a two vector self-organization mechanism, named behavioural and structural self-organization. The structural self-organization, detailed in this paper, operates in a macro-level and is related to the relations between holons and holarchies formation.

$\mathrm{ADACOR}^{2}$ by being proposed to work in highly dynamic systems is supported with learning mechanisms allowing the dynamic discover of new ways to evolve. Additionally, by giving autonomy to these entities, they can display some nervous- 
ness, making the system to become more unstable. The idea is to push the system to its limits, by enhancing the self-organization and chaotic principles, but maintaining the system under control, by actuating in the nervousness control.

The structural self-organization mechanism was tested in a production cell, inspired in the real AIP cell, where resources have the possibility to shift their position in the shop-floor. The achieved preliminary results show that enabling the structural self-organization mechanisms a performance increase is achieved.

Future work will be devoted to improve the algorithms considered in the structural self-organization mechanism and to merge the two self-organization principals in $\mathrm{ADACOR}^{2}$.

\section{$7 \quad$ References}

1. Wooldridge, M.J.: An introduction to multiagent systems. John Wiley \& Sons, Chichester, U.K (2009).

2. Koestler, A.: The Ghost in the Machine. Arkana Books (1969).

3. Leitão, P.: Agent-based Distributed Manufacturing Control: A State-of-the-art Survey. Proceedings of the International Journal of Engineering Applications of Artificial Intelligence. 22, n. 7, 979-991 (2009).

4. Leitao, P., Marik, V., Vrba, P.: Past, Present, and Future of Industrial Agent Applications. IEEE Transactions on Industrial Informatics. 1-1 (2012).

5. Leitão, P., Restivo, F.: ADACOR: A Holonic Architecture for Agile and Adaptive Manufacturing Control. Computers in Industry. 57 (2), 121-130 (2006).

6. Parunak, H.V.D., Brueckner, S.A., Matthews, R., Sauter, J.: How to calm hyperactive agents. Presented at the (2003).

7. Hadeli, Valckenaers, P., Verstraete, P., Germain, B.S., Brussel, H.: A Study of System Nervousness in Multi-agent Manufacturing Control System. In: Brueckner et al (ed.) Engineering Self-Organising Systems. pp. 232-243. Springer Berlin Heidelberg (2006).

8. Barbosa, J., Leitão, P., Adam, E., Trentesaux, D.: Nervousness in Dynamic Selforganized Holonic Multi-agent Systems. Highlights on Practical Applications of Agents and Multi-Agent Systems. pp. 9-17. Springer Berlin Heidelberg (2012).

9. Borgo, S., Leitão, P.: Foundations for a Core Ontology of Manufacturing. In: Sharman et al. (ed.) Ontologies. pp. 751-775. Springer US, Boston, MA.

10. Panait, L., Luke, S.: Cooperative Multi-Agent Learning: The State of the Art. Autonomous Agents and Multi-Agent Systems. 11, 387-434 (2005).

11. Pach, C., Bekrar, A., Zbib, N., Sallez, Y., Trentesaux, D.: An effective potential field approach to FMS holonic heterarchical control. Control Engineering Practice. 20, 1293-1309 (2012).

12. Bellifemine, F.L., Caire, G., Greenwood, D.: Developing multi-agent systems with JADE. John Wiley, Chichester, England; Hoboken, NJ (2007).

13. Friedman-Hall, E.: Jess in action. Manning; Pearson Education, Greenwich, Conn.; London (2003). 